https://doi.org/10.24201/aap.2021.323

INFORME

\title{
Vietnam 2020: un año con pocos éxitos, muchos retos e incertidumbre
}

\section{Vietnam 2020: A Year with few Successes, many Challenges, and Uncertainty}

\author{
MARICELA MIREYA REYES LÓPEZ \\ https://orcid.org/0000-0002-5419-4002 \\ Universidad de Colima, México
}

Recepción: 29 de octubre de 2020

Aceptación: 15 de diciembre de 2020

Resumen: Desde el inicio de 2020, el panorama de Vietnam parecía prometedor, interna y externamente. Iniciaba su desempeño en el doble papel de ser presidente de la Asociación de Naciones del Sureste Asiático (ANSEA) y de ser miembro no permanente en el Consejo de Seguridad de las Naciones Unidas (ONU). Asimismo, los lazos económicos y políticos con actores de la región, como la Unión Europea, Estados Unidos y Japón, dejaban entrever las posibilidades de mejorar como socio comercial de calidad y como socio económico y político. Algunas actividades se ensombrecieron con la covid-19, cambiando las prioridades a un segundo plano. No obstante, Vietnam pudo superar dificultades en medio de la pandemia. El presente reporte expone acontecimientos de política exterior, de relación comercial, de representación y de aspectos socioeconómicos que Vietnam recorrió el año 2020.

Palabras clave: Vietnam-ANSEA; Vietnam-Consejo de Seguridad; acuerdo de libre comercio; Mekong-seguridad humana; notas diplomáticas. 
Abstract: From the beginning of the 2020, internally and externally the outlook for Vietnam was promising. It began with its performance in a double role as president of the Association of Southeast Asian Nations (ASEAN) and a non-permanent member of the United Nations (UN) Security Council. Likewise, the economic and political ties with actors in the region, such as the European Union, the United States and Japan, hinted its possibilities of improving as a quality trading partner and as economic and political partner. Some activities were overshadowed by Covid-19, changing the priorities to second place. However, Vietnam was able to overcome difficulties during the pandemic. This report presents events of foreign policy, trade relations, representation, and socioeconomic aspects that Vietnam went through in 2020 .

Keywords: Vietnam-ANSEA; Vietnam-Security Council; free trade agreement; Mekong-human security; diplomatic notes.

\section{ForTALECIMIENTO DE LOS VÍNCULOS COMERCIALES DE VIETNAM CON EUROPA}

Sin duda, uno de los logros de Vietnam en 2020 es la firma del Acuerdo de Libre Comercio Unión Europea-Vietnam (EVFTA, por sus siglas en inglés). No fue sencillo lograr este Acuerdo por varias razones. Una, porque la Unión Europea (UE) antepone el tema de los derechos humanos a cualquier iniciativa de lazo comercial con cualquier país, y Vietnam no es excepción. Según Amnistía Internacional, el acuerdo es claro al solicitar al gobierno vietnamita permitir el establecimiento de sindicatos independientes, progresar en un marco legal sobre derechos laborales y ratificar convenciones de la Organización Internacional del Trabajo (Amnistía Internacional s. f.). No obstante que esta solicitud todavía es un reto, las Naciones Unidas reconocen que el gobierno de Vietnam ha tenido algunos avances en materia de derechos humanos porque ratificó los principales tratados internacionales en estos asuntos, incluida la Convención contra la tortura y otros tratos o penas crueles, inhumanas o degradantes y la relativa a los Derechos de las Personas con Discapacidad (DAN 2020). La violación a los derechos humanos sería una de las razones que pondría en riesgo el EVFTA. 
Otras solicitudes del acuerdo incluyen garantías vinculantes con los temas de protección del clima y de derechos laborales.

Una segunda razón que generó altibajos en esas negociaciones fueron las cuestiones sociales y la presión sobre Vietnam para liberalizar las barreras no arancelarias, las reglas de origen, así como las regulaciones técnicas y sanitarias. Pero a diferencia de los acuerdos comerciales de la UE con países de África, el Caribe y el Pacífico, el EVFTA no contiene protecciones para la industria naciente y requiere "medidas de salvaguardia bilaterales recíprocas estándar para proteger a los productores nacionales". La disputa de Vietnam con la Unión Europea por las medidas antidumping aumentó la sensibilidad de esta cuestión (Hoang 2020), y también el asunto de la propiedad intelectual, problema que en Vietnam no se ha podido eliminar pues existe gran cantidad de imitaciones de productos y copias locales.

Retomando el aspecto comercial del acuerdo, se puede decir que beneficiará a Vietnam al aumentar sus ventas con países europeos, iniciando con un incremento de $20 \%$ de las exportaciones este año, según fuentes de gobierno. No debe olvidarse que el país ha sido atractivo para las inversiones desde hace varios años, y los europeos saben que Vietnam tiene una economía dinámica y gente joven con porvenir que puede asegurar una gran confianza para inversionistas. Se facilitará el flujo de personas que soliciten visas o permisos de trabajo.

El libre comercio entre ambas partes no sólo diversificará los productos exportados, sino que podría eliminar todo arancel en diez años. Es tan ambicioso el acuerdo que se menciona la eliminación casi de inmediato de $86 \%$ de los aranceles que se aplican ahora a productos vietnamitas (Hoang 2020). Los aranceles más bajos sobre los productos vietnamitas con destino a la UE darán a Vietnam una ventaja sobre los competidores miembros de la ANSEA y de China. Vietnam exporta a la UE equipos de telecomunicaciones, ropa y productos alimenticios, y la UE exporta a Vietnam maquinaria y equipos de transporte, productos químicos y productos agrícolas. El acuerdo establece también que compañías europeas podrán participar en las licitaciones públicas convocadas por el gobierno vietnamita y varias ciudades, entre ellas Hanoi (Parlamento Europeo 2020).

En términos generales, Vietnam comercializará directamente con todos los países de la UE a partir del $1^{\circ}$ de agosto, cuando entre en vigor el EVFTA. Seguramente España, Italia, 
Francia y Alemania, países con quienes Vietnam ha tenido importantes intercambios, podrán hacerlo bajo los esquemas preferenciales. Vietnam es el segundo socio económico más importante de la UE dentro de la ANSEA, y con retos y oportunidades se espera que, a mediano y largo plazos, se pueda hablar de un acuerdo realmente positivo para ambas partes.

\section{Prestigio REgIONAL COMO PRESIDENTE}

\section{DE LA ANSEA 2020 (ANFITRIÓN)}

Debido a que en 2020 se revisa el establecimiento de la comunidad de la ANSEA 2015-2025, Vietnam eligió titular la reunión cumbre "ANSEA cohesiva y receptiva”, como tema-eslogan de su presidencia. Lo "cohesivo" representa la idea de defender la unidad, la solidaridad y la fuerza interna de la asociación, aumentar su conectividad económica, profundizar sus valores e identidades, mejorar la eficiencia del aparato del bloque y promover su cooperación en la comunidad mundial. "Responsivo" refleja las necesidades de la ANSEA de aumentar su proactividad, creatividad y capacidad de respuesta a las oportunidades y desafíos de la situación mundial y regional (ASEAN Vietnam s. f.).

El 6 de enero el primer ministro vietnamita, Nguyen Xuan Phuc, mencionó en su discurso de apertura cinco prioridades en las que se fundamenta el título: contribuir a la paz, a la seguridad y a la estabilidad regionales fortaleciendo la solidaridad y la unidad de la ANSEA; intensificar la conectividad regional mediante el uso de tecnologías digitales y novedosas; promover las identidades y los valores compartidos de la asociación; fortalecer las alianzas mundiales para la paz y el desarrollo sostenible, y mejorar su capacidad de respuesta y su eficacia operativa (Tran 2020). Respecto de la segunda prioridad, de intensificar la conectividad regional, el brote de covid-19 perturbó las cadenas de suministro globales y regionales, por lo que la conectividad no logra concretarse.

Algunas prioridades se están viendo modificadas, y han sido canceladas actividades de las más de 300 conferencias o encuentros planeados desde 2019, además de actividades conmemorativas del 25 aniversario de la membresía de Vietnam a la ANSEA y la promoción de interacciones regionales. 
Obviamente, los encuentros ministeriales y de grupos se orientaron a discutir el tema covid-19. Sólo se resumen aquí esas reuniones dentro de ANSEA. Vietnam trabajó de forma estrecha en la colaboración con los miembros de la asociación para ayudar a hacer frente a los resultados negativos generados por propagación del SARS-CoV-2. Se fueron sucediendo las distintas reuniones durante todo el año. El 14 de febrero, Vietnam emitió una declaración desde la presidencia de ANSEA sobre la respuesta colectiva de los Estados miembros al brote de covid-19, destacando la importancia de la solidaridad, y promovió la cooperación en múltiples niveles. El 31 de marzo se celebró en Hanoi la teleconferencia del Grupo de Trabajo del Consejo Coordinador de la ANSEA sobre Emergencias de Salud Pública para que los Estados miembros compartieran información sobre su situación y la implementación de medidas de control. A nivel de ministros de Relaciones Exteriores, el 20 de marzo y el 9 de abril Vietnam presidió las sesiones del Consejo de Coordinación de la ANSEA y la de ministros de Relaciones para discutir formas de fortalecer la colaboración entre el grupo y sus socios.

Siguiendo el espíritu de una "ANSEA cohesiva y receptiva”, Vietnam organizó el 14 de abril una cumbre especial sobre coronavirus e instó a los Estados miembros a permanecer unidos y actuar con decisión en respuesta a la pandemia. Los líderes acordaron crear un Fondo de Respuesta Covid-19 ANSEA y reservas regionales de suministros médicos.

En otra instancia, Vietnam promovió la cooperación de ANSEA con países de todo el mundo, como se evidencia en la Reunión de Ministros de Relaciones Exteriores de ANSEAChina sobre cooperación para responder a la covid-19, llevada a cabo el 20 de febrero. El ministro de Relaciones Exteriores de China, Wang Yi, informó al organismo de la situación en Wuhan y en otras partes de China. El bloque confirmó su apoyo a China en la lucha contra la enfermedad. Otra evidencia fue la reunión del 20 de marzo, cuando Vietnam presidió la teleconferencia ministerial ANSEA-Unión Europea sobre cooperación contra la pandemia. Se acordó aumentar el intercambio de información, de experiencias y consulta de políticas en el diagnóstico, tratamiento y producción de vacunas.

Paralelamente, el 29 de marzo Vietnam fue invitado a la cumbre de emergencia en línea del G20 sobre covid-19. Además de compartir la experiencia de control de la enfermedad en Vietnam, el primer ministro, Nguyen Huan Phuc, destacó la importancia de 
la solidaridad, cooperación y colaboración a nivel mundial y regional, y refirió que la lucha contra la pandemia debía facilitar también la cooperación comercial y la inversión. El 14 de abril, Vietnam también presidió la Cumbre Especial ANSEA + 3 sobre covid-19. Los socios de diálogo China, Japón y Corea del Sur reconocieron la importancia de la cooperación ANSEA +3 y de los mecanismos existentes para abordar los desafíos de salud pública.

A pesar de que la cumbre Estados Unidos-ANSEA se programó inicialmente para mediados de marzo, ésta se pospuso, y Vietnam celebró el $1^{\mathrm{o}}$ de abril la videoconferencia interinstitucional de alto nivel ANSEA-Estados Unidos sobre cooperación para contrarrestar la covid-19. En esa ocasión se reiteró el valor de la asociación estratégica ANSEA-EU para enfrentar los desafíos sin precedentes de la pandemia. El éxito de la reunión llevó a realizar el 23 de abril la videoconferencia especial ministerial ANSEA-EU sobre covid-19, en la que participó el secretario de Estado de EU, Mike Pompeo. El viceprimer ministro y ministro de Relaciones Exteriores de Vietnam, Pham Binh Minh, agradeció a Estados Unidos un apoyo financiero de 19 millones de dólares otorgados (Tran 2020).

Como se observa, el tema covid-19 acaparó la discusión en casi todas las reuniones de la ANSEA. Algunos objetivos planeados por la presidencia en turno tendrán que retomarse por Brunéi como la próxima estafeta. De hecho, ya el sultanato bruneiano dispuso algunos preparativos presupuestales para 2021.

Sin duda, el resultado de la reunión ministerial de ANSEA fue la firma de la Asociación Económica Integral Regional (RCEP, por sus siglas en inglés) el 15 de noviembre, proceso que inició en 2012 en Camboya y que ahora culmina en Vietnam 2020. La firma de los diez miembros de la ANSEA, más Australia, China, Corea del Sur, Japón y Nueva Zelanda, en suma 15 actores, representa la zona de libre comercio más grande del mundo. Los efectos geopolíticos no son claros, pero habría que pensar en algunos puntos: 1) Es un acuerdo multilateral, con presencia de China y ausencia de Estados Unidos; 2) El PIB mundial de la RCEP, o de sus 15 miembros, representa 30\%, sólo mientras Estados Unidos decide si vuelve o no al Tratado Integral y Progresista de Asociación Transpacífico (CPTPP, por sus siglas en inglés, Comprehensive and Progressive Agreement for Trans-Pacific Partnership, antes TPP, Trans-Pacific Partnership), con lo que ese indicador aumentaría (Fabry 2020); 3) La reacción de la Comunidad Europea, con la que hay tratados bilaterales y con la propia ANSEA; 
4) Habría que analizar en qué términos se reducirán los aranceles en la RCEP y en el CPTPP para conocer las conveniencias de cada uno.

Por supuesto, dos de los temas que no se discutieron a profundidad fueron: 1) el código de conducta largamente buscado por la disputa del Mar de China Meridional y 2) el acuerdo sobre los recursos hídricos en la gestión del Río Mekong.

Como señal de la creciente resistencia al oportunismo estratégico de China por la covid-19, el bloque de ANSEA, antes discreto, ha articulado una postura más dura sobre la intensificación de las disputas del Mar de China Meridional. La ANSEA pronunció categóricamente que la Convención de las Naciones Unidas sobre el Derecho del Mar (UNCLOS, por sus siglas en inglés) de 1982 es "la base para determinar los derechos marítimos, los derechos soberanos, la jurisdicción y los intereses legítimos sobre las zonas marítimas". Es la primera vez que el organismo regional identifica explícitamente la UNCLOS como la única base legal para resolver disputas marítimas y territoriales en la región. Ahora, contrariamente, los países del Sudeste Asiático rechazan abiertamente cualquier propuesta de China debido, probablemente, a los esfuerzos decididos del actor que preside rotatoriamente a ANSEA, Vietnam, país que ha tratado de movilizar la unidad regional en las disputas a pesar de la irrupción que hace la pandemia en la celebración de cumbres regionales.

Con un tuit del 29 de junio la administración Trump acogió calurosamente la última declaración de la ANSEA sobre el Mar de China Meridional, cuando el secretario de Estado de Estados Unidos, Mike Pompeo, escribió: “Estados Unidos da la bienvenida a la insistencia de los líderes de la ANSEA en que las disputas del Mar de China Meridional se resuelvan de conformidad con el derecho internacional" (Heydarian 2020a).

\section{MAR de China Meridional, nOtas VERBAles O “Dimes Y DiRETES”; EJERCICIOS MILITARES EN ALTA MAR}

El tema recurrente del Mar de China Meridional, ${ }^{1}$ característico de la política exterior de Vietnam y China por más de cuatro décadas, no parece tener fin. En realidad, éste resurge

\footnotetext{
${ }^{1}$ La disputa del Mar del Sur Meridional no es sólo una disputa sobre la propiedad de islas, arrecifes, atolones y bancos de arena, también es una disputa sobre los derechos marítimos a propósito de cuánta zona marítima tienen esos territorios
} 
con frecuencia como pretexto sin ánimos de solución. Desde el año pasado, cuando Estados Unidos hizo otra más de las denuncias públicas en contra de lo que llama "imperialismo chino", esta denuncia se concretó en la firma de un acuerdo entre Estados Unidos con Vietnam.

En 2020 una de las particularidades sobre el asunto del Mar de China Meridional es una serie de denuncias verbales que hicieron reclamantes y no reclamantes. En diciembre de 2019 Malasia presentó ante la Comisión de Límites de la Plataforma Continental (CLPC) información sobre su plataforma continental ampliada de lo que "le corresponde" en la parte norte del Mar de China Meridional. Ello desencadenó un debate entre los reclamantes. Varios comunicados se generaron por parte de China, Filipinas, y Vietnam a manera de notas verbales, la de Vietnam emitida el 30 de marzo de 2020 para protestar contra la posición de China.

En detalle, la nota verbal 22/HC-2020 de la Misión Permanente de la República Socialista de Vietnam ante Naciones Unidas explica la posición del gobierno vietnamita en la disputa del Mar de China Meridional y objeta los derechos históricos de China en estas aguas y cualquier otro reclamo marítimo que exceda los límites establecidos en la Convención de Naciones Unidas sobre el Derecho del Mar (Convemar). La nota implica que todas las características de marea alta en las islas Spratly y en las Paracel son rocas que no generan ningún derecho a una ZEE o plataforma continental (Diep 2020).

Por su parte, Australia presentó otra nota verbal el 23 de julio al secretario general de la ONU en la que expuso su posición sobre las reclamaciones de China en el Mar de China Meridional. La nota australiana fue una respuesta a la presentada por Malasia en 2019. No está claro por qué Australia y Estados Unidos hicieron declaraciones en este momento en particular, aparte de agregar otra dimensión a la creciente rivalidad con China. Tampoco hubo nada nuevo en las declaraciones de China a la CLPC (Bateman 2020).

(disputados) legalmente. Los derechos marítimos están asociados con los derechos y la jurisdicción sobre los recursos naturales y las actividades económicas, como la pesca, la explotación de hidrocarburos, las islas artificiales y la investigación científica marina. Debido a su importancia para el crecimiento económico, en las últimas décadas se ha vuelto crítica la disputa sobre los derechos marítimos. 
A principios de junio, la embajadora de Estados Unidos ${ }^{2}$ ante las Naciones Unidas, Kelly Craft, presentó una nota verbal a la ONU en la que rechazó los reclamos marítimos de China como "incompatibles con el derecho internacional". "[Estados Unidos] se opone al reclamo de China de 'derechos históricos' en el Mar de China Meridional en la medida en que el reclamo exceda los derechos marítimos que China podría afirmar de conformidad con el derecho internacional reflejado en la UNCLOS", dijo la embajadora (Heydarian 2020a).

Por último, Indonesia presentó en mayo su carta verbal ante Naciones Unidas en la que cuestionó directamente las reclamaciones de China. "Indonesia reitera que el mapa de línea de nueve puntos que implica reclamos de derechos históricos carece claramente de base legal internacional y equivale a alterar la Convención de las Naciones Unidas sobre el Derecho del Mar de 1982” (Heydarian 2020a).

Los ejercicios militares llevados a cabo en el Mar de China Meridional por parte de China no son particularidades de 2020, pero sí son un hecho repetido. En los primeros días de junio, un barco patrullero de la Guardia Costera china embistió un barco pesquero vietnamita que navegaba en las islas Paracel. Esta actitud no es rara dado el manual de pesca de Beijing, pero es importante porque es la primera vez que China aplica su prohibición de pesca unilateral en el Mar de China Meridional. Hace años Beijing anunció la prohibición anual, la cual ahora provocó una fuerte protesta por parte de Filipinas y Vietnam. Pero China no había aplicado la regla a los buques extranjeros sino hasta este año.

Simultáneamente, otro buque de inspección del gobierno chino, Haiyang Dizhi 4, maniobró alrededor de la ZEE vietnamita en un movimiento que, se cree, pretende presionar a Hanoi para que no siga realizando exploración de petróleo y gas en esta área.

Un asunto que se ha dejado de mencionar es el establecimiento de la prefectura de la ciudad de Sansha. ${ }^{3}$ Esto no es nuevo porque desde 2012 el Consejo de Estado Chino estableció esa ciudad a nivel de prefectura perteneciente a la provincia de Hainan. La

\footnotetext{
${ }^{2}$ Debido a que Estados Unidos no es parte de la UNCLOS de 1982, fue restringida su capacidad para presentar comunicaciones a la CLCS - un organismo establecido bajo la UNCLOS.

${ }^{3}$ Sansha se encuentra al sur de la isla china de Hainan. Desde 2012 se ha convertido en una ciudad urbana. Hoy tiene escuela, atrajo inversión corporativa considerable, tiene infraestructura portuaria, plantas de desalinización de agua de mar, estableció una industria turística en auge, diversificó su economía, mejoró la logística y las telecomunicaciones, construyó viviendas permanentes y promovió la residencia normalizada. Sansha también tiene sistemas de inteligencia, vigilancia y reconocimiento avanzados. Véase Haver 2020.
} 
discusión gira en torno a que la jurisdicción de Sansha abarca más de 280 islas y bancos de arena, lo que equivale a casi 800000 millas cuadradas de zona de mar y tierra. Esto incluye parte de los reclamos de China dentro de su línea de nueve puntos, incluidas las islas Paracel y las Spratly reclamadas por Vietnam. El 18 de abril China anunció que establecería el distrito administrativo de Sansha, con lo que amplía su jurisdicción en varias islas y atolones y confirma ser propietario de ellas (Haver 2020). La reacción de Vietnam fue oponerse a esta posición porque considera que Nansha (Spratly) es parte del archipiélago vietnamita de Truong Sa, y Xisha (Paracel) en el archipiélago vietnamita de Hoang Sa, donde se localiza Sansha.

\section{SEguridad humana EN EL DELTA DEL MEKONG EN Vietnam}

Es probable que la seguridad humana relativa al delta del Mekong, no sea un tema universal como debiera. No obstante, las consecuencias negativas, y el deterioro en áreas como medio ambiente, pobreza, clima, producción de alimentos, salud, etc., sí llegarán a trastocar, directa o indirectamente, a todos los seres humanos.

\section{Abundancia y riqueza para Vietnam}

La extensión más baja del Río Mekong es lo que forma el delta y se encuentra en Vietnam. ${ }^{4}$ El delta del Mekong de Vietnam es una región plana y baja que favorece la anegación del suelo durante la época de lluvias. Se extiende por poco más de 40000 kilómetros cuadrados, de los cuales, en promedio 24000 se utilizan en la agricultura y 4000 para la silvicultura. En esta área viven aproximadamente 21 millones de personas (Koema 2020). El delta del Mekong de Vietnam tiene una importante producción agrícola de exportación del país,

\footnotetext{
${ }^{4}$ Los vietnamitas llaman al delta la región del Cuu Long, que quiere decir "Nueve Dragones", porque justamente está dividido en nueve grandes brazos acuíferos que fluyen hasta desembocar en el mar. Llamado Nam Bo por los vietnamitas y Conchinchina por los franceses, el sur de Vietnam, incluida el área del delta, se extiende desde Truong Son hasta la punta de la península de Ca Mau (parte sur de Vietnam). Los afluentes de los nueve ríos cruzan diez de las trece provincias del delta, desde la provincia de Long An hasta la provincia de Soc Trang. Las localidades por donde cruza el Río Mekong en Vietnam y sus afluentes son áreas que reciben agua dulce durante todo el año, y al mismo tiempo hay terrenos con muy rica sedimentación proveniente de las fuentes de agua dulce de las partes altas.
} 
incluida la de arroz, para exportación y para consumo interno. Asimismo, es considerable la producción de fruta, de pesquerías y de acuacultura.

\section{Impactos negativos en el medio ambiente por la mano del hombre} y por cambio climático en la agricultura

Según expertos, la producción de arroz en el delta del Mekong debiera reducirse porque probablemente la región va a enfrentar escasez de agua en las próximas décadas. El profesor Vo Tong Xuan, experto en agricultura, dice que los campesinos no saben cómo utilizar el agua económicamente y cada hectárea de tierra labrada consume más de 20000 metros cúbicos de agua. Esto significa que 3.8 millones de hectáreas arroceras del delta del Mekong necesitarán más de 76 millones de metros cúbicos de agua anuales (VietNamNet 2017). Aunque se necesita mucha agua para los arrozales, el problema se agudiza con los efectos del cambio climático y los proyectos de hidroenergía construidos río arriba que agotan el agua antes de que ésta llegue a la región de gran producción. La economía de la región depende del agua, pero las autoridades provinciales vietnamitas no han mapeado algún plan coordinado para este asunto, dicen expertos (VietNamNet 2017).

Vietnam se encuentra entre los países más vulnerables a los efectos del cambio climático en el mundo, sufrirá consecuencias graves más que otros. Si no se toman medidas drásticas en este momento, puede revertirse el progreso que ha hecho en producción agrícola, reducción de pobreza y diversificación en exportaciones al mundo.

Desde 2019 los efectos ambientales han hecho estragos en el norte y sur del país. El sur ha sufrido este año severas sequías como lluvias torrenciales. Desde abril hasta octubre se registraron inundaciones con consecuencias graves para la población y la producción. Según el informe semestral de la Oficina de Estadística de Vietnam (GSO), la catástrofe natural en los primeros seis meses del año causó 40 muertos y 133 heridos; dañó más de 118000 hectáreas de arroz y casi 35800 hectáreas de otros cultivos; 1167 casas colapsaron y casi 64000 se dañaron. Se estimó una pérdida total de activos en casi 4 billones de dong. La sequía y la intrusión salina en el centro y delta del Mekong causaron pérdidas en unas 90000 hectáreas de arroz y 22500 hectáreas de otros cultivos; el valor total de los daños ascendió a 3.6 billones de VND. El valor total de los activos causados por desastres naturales 
en el primer semestre de 2020 se estimó en más de 4.8 billones de VND, 10 veces más alto que el valor dañado en el mismo periodo en 2019 (GSO 2020c).

En el norte, en junio y julio, la región registró una de las más largas temporadas de olas de calor que no sucedía desde la década de 1970. En una gran extensión que cubren las provincias de Lang Son, Bac Ninh, Bac Giane, Hai Duong, Hung Yen, Ha Han y Ninh Binh se registró la ola de calor que duró más de dos días con 36 hasta $39^{\circ} \mathrm{C}$ (Quy 2020). La situación generó escasez del agua de riego e incendios forestales. Algunas oficinas e instituciones cuantifican costos a futuro. De acuerdo con el Panel Intergubernamental sobre Cambio Climático, para 2030 Vietnam puede perder 5.7 por ciento del PIB debido al calor excesivo que le costarán $\$ 85000$ millones en pérdida de productividad, pues el clima hace imposible trabajar al aire libre en los meses de verano (Quy 2020).

Para argumentar la seriedad de los efectos del cambio climático en el delta del Mekong se publicaron dos estudios que fueron cuestionados por la mención de algunos "responsables" de ser parte del problema. Uno de los informes pertenece a Eyes on Earth y el otro al Stimson Center.

La publicación de Eyes on Earth se refiere a los niveles de agua medidos en Chiang Saen, en el norte de Tailandia, que arrojó información sobre lo que está sucediendo en la cuenca china del Lancang (así nombran los chinos al Mekong). Luego, la publicación del Stimson Center afirmó que el estudio Eyes on Earth es una prueba definitiva de que la política china de gestión del agua causó la sequía. Las afirmaciones son ya parte del repetido discurso que acusa la sabida creciente influencia de China para alterar los flujos de agua que corren hacia el bajo Mekong.

Se cuestiona que el estudio de Eyes on Earth no proporciona una prueba definitiva de la responsabilidad de China, pero sí representa una ilustración novedosa del potencial de la tecnología de imágenes por satélite para trascender las fronteras nacionales y revelar de forma independiente las condiciones hidrológicas en la cuenca. Los principales hallazgos técnicos del estudio de Eyes on Earth subrayan que el flujo en Chiang Saen comenzó a diferir de las condiciones naturales en 2012, cuando la represa de Nouzhadu en la provincia china de Yunnan comenzó a llenarse. Si bien los estudios no proporcionan información comprobada, e incluso resulta contradictoria, se ha despertado nuevamente la atención sobre una baja en 
los caudales de agua del Mekong y los efectos de la mano del hombre que, combinados con los efectos del cambio climático, ponen en riesgo una fuente de alimento de millones de personas y altera el ecosistema.

Mientras tanto, el gobierno vietnamita indicó en una conferencia de prensa que, para limitar los impactos negativos del cambio climático en los resultados de la producción agrícola, es necesario ajustar los planes de producción, cambiar la estructura de los cultivos y adaptarlos a cada región o cambiar de cultivo de producción para aprovechar las ventajas de las provincias. Asimismo, indicó que deben brindarse soluciones rápidas para el sostenimiento del mercado frente al problema covid-19, que ha reducido la demanda de consumo interno y externo (GSO 2020a).

Por otro lado, en Camboya se ha detectado un efecto negativo en el Lago Tonle Sap, que también recibe aguas de Mekong. Cada año el lago se llena y se descarga gracias a la inundación que provoca la estación de lluvias durante el monzón, y poco a poco se descarga durante la estación de secas. Aunque no llueva, el agua dulce elimina el agua de mar. Generalmente el Tonle Sap completa un proceso de drenado en marzo, y durante este tiempo hay agua salada por un mes hasta la llegada del monzón, proceso que se repite cada año (Osborne 2020). Sin embargo, en 2020 el Tonle Sap no se llenó, y se redujo el nivel de agua, en tanto que la sal se quedó por más meses. Es importante mencionar el problema en el Lago Tonle Sap porque la poca profundidad impide el desarrollo natural de los peces, y los pescadores obtienen menor producción (Fawthrop 2020).

Siguiendo el tema de exceso de construcción de represas, ambientalistas dijeron que represar el Mekong — que se ha hecho por años— tiene costos ambientales elevados no sólo para Vietnam, sino para Tailandia, Laos y Camboya. Contrariamente a la idea general que se tiene de un delta del Mekong fértil, la región sufre cada vez más de intrusión salina y cambios en los patrones del monzón. Las inundaciones sufridas y la degradación de las pesquerías, resultado de la construcción de represas río arriba, ponen en peligro la economía del delta, especialmente en un año en el que el gobernante Partido Comunista se concentra en reequilibrar su liderazgo. El partido-Estado necesita demostrar que está defendiendo los intereses de delta (Brown 2020). 
China niega que las represas que construye causen las sequías y está en desacuerdo con las referencias y acusaciones que hacen los informes. Cualquiera que sea el argumento, están en juego el suministro de alimentos y los medios de vida de millones de personas. Un estudio presentado por el Departamento de Agricultura de Estados Unidos pronostica que, debido a la sequía y a la intrusión de agua salada, los rendimientos de la producción de arroz de 2020 en Vietnam caerían 3.3\% de acuerdo con las estimaciones anteriores, dejando la cosecha $0.9 \%$ más baja para el año. La Comisión del Río Mekong (MRC, por sus siglas en inglés) advirtió sobre las consecuencias "graves" de la interrupción de la captura de peces, especialmente porque se estima que, de los 60 millones actuales, la población del bajo Mekong podrá aumentar a 100 millones de personas para 2025. También pronosticó una posible caída de 40\% en las capturas de pescado hasta 2020, y de 80\% menos para 2040. El informe muestra que, a medida que se pronostica que las poblaciones aumentarán a lo largo del Mekong, es probable que las poblaciones de peces colapsen debido a una combinación de represas, pesca ilegal provocada por la escasez y el cambio climático (Zhou 2020).

En una conferencia de prensa del gobierno vietnamita se hizo evidente la intensidad de los problemas ambientales que padece el país, y llamó a la necesidad de atender la situación meteorológica para prevenir de forma proactiva los desastres naturales, advertir inundaciones, deslizamientos de tierra, impactos de la sequía e intrusión salina para minimizar los daños a la producción y la vida de las personas. Se hizo el llamado a implementar efectivamente políticas en materia de seguridad social, laboral y empleo para asegurar que las personas que enfrentan riesgos y desastres naturales obtengan apoyo oportuno, superen dificultades y estabilicen sus vidas. Reforzar la labor de velar por el orden y la seguridad del tráfico, proteger el medio ambiente, prevenir incendios y explosiones fueron también expresados a manera de urgencia por parte del gobierno (GSO 2020b). 


\section{POLÍTICA EXTERIOR: VIETNAM, MIEMBRO NO PERMANENTE del Consejo de Seguridad de la ONU (CSNU) PARA 2020-2021 Y RELACIONES CON OTROS PAÍSES}

La política exterior de Vietnam se caracterizó en 2020 por el logro obtenido como miembro no permanente del Consejo de Seguridad, y, además del acuerdo firmado con la Unión Europea ya mencionado, bilateralmente fortaleció lazos con Estados Unidos, Japón y otros.

Como miembro no permanente, seguramente la situación del trabajo y reuniones en línea han sido arduas y diferentes, como ha sido el trabajo que realiza en línea como presidente de ANSEA 2020. Representantes de muchos países de la ONU enviaron felicitaciones a Vietnam por convertirse en un miembro no permanente del CSNU para 20202021, con 192 votos del total de 193 países miembros de la ONU. Destacan dos iniciativas vietnamitas propuestas para debatir en la mesa de discusión del Consejo de Seguridad: 1) Impulsar el cumplimiento de la Carta de las Naciones Unidas en el mantenimiento de la paz y la seguridad internacional, y 2) coordinar una reunión sobre la cooperación entre las Naciones Unidas y la ANSEA.

La evaluación que hizo el viceministro de Relaciones Exteriores de Vietnam en torno al papel desempeñado por el país en el primer semestre del año como miembro no permanente del Consejo de Seguridad es muy ilustrativa porque indica actividades diversas: junto con los otros no miembros, Vietnam solicitó que el Consejo de Seguridad realizara una videoconferencia con el tema covid-19, y, se dice, fue la primera de su tipo efectuada a inicios de abril; propuso celebrar una mesa de diálogo de alto nivel entre la ANSEA y la ONU sobre desarrollo sostenible en la cumbre entre las dos partes en octubre de 2020 (Huyền y Phương 2020).

Entre las respuestas a favor como Estado miembro no permanente del Consejo de Seguridad, el voto de Vietnam ha sido para diferentes causas: promoción de solución de una política integral duradera que dé respuesta a los desafíos de seguridad y de inestabilidad socioeconómica en Siria; apoyo a la independencia, soberanía e integridad del territorio de Chipre; apoyo en la implementación de los acuerdos de paz en Colombia, entre otros. 


\section{Vietnam y Estados Unidos}

Puede decirse que entre ambos países existen intereses económicos y políticos. El interés de Vietnam es económico, y el de Estados Unidos es político. Se espera que los lazos económicos entre Vietnam y Estados Unidos se fortalezcan. Estados Unidos es el segundo socio comercial más grande de Vietnam, detrás de China. El comercio se ha expandido incluso con la crisis covid-19 pues aumentó a \$26 000 millones de dólares en los primeros cinco meses del año, casi $8 \%$ más que en el mismo periodo del año pasado (Hutt 2020a). Algunos analistas dicen que el país más beneficiado de la guerra comercial entre Estados

Unidos y China es Vietnam debido al traslado industrial que algunas empresas extranjeras en China están haciendo hacia Vietnam.

En el ámbito político, una de las razones del intenso vínculo entre Vietnam y Estados Unidos es que este último es el oponente más fuerte de los países de la región contra el expansionismo chino, especialmente el que representa en el Mar de China Meridional, donde Pekín y Hanoi se disputan territorio. Hutt refiere que Estados Unidos significa para Vietnam un colchón de superpotencia y un protector potencial contra China, su ocupante histórico y una amenaza percibida desde hace mucho tiempo (Hutt 2020a).

\section{Vietnam y Japón}

Vietnam mantuvo un acercamiento de amistad evidente con el primer ministro japonés Shinzō Abe, desde que inició hasta su dimisión en septiembre. En medio de la creciente postura china, Japón respaldó la postura de Vietnam sobre la gestión de disputas en el Mar de China Meridional y subrayó la importancia de respetar el derecho internacional y el apoyo de seguridad naval.

Con el nuevo primer ministro de Japón, Yoshihide Suga, la relación con Vietnam avanzó mejor que nunca, pues siendo el primer país del Sureste de Asia que el ministro japonés visitó, tuvo un acierto con la firma de un acuerdo para venta de equipo y tecnología militar japonesa, en un marco del ya repetido eslogan "cooperación en defensa y seguridad" en la región. El equipo ayudará a mejorar la capacidad de vigilancia de Vietnam en el Mar de China Meridional. Básicamente es para contrarrestar la influencia china en la región pues 
también Vietnam, que ya es beneficiario de las patrullas japonesas, firmó un acuerdo para comprar seis patrullas adicionales de la guardia costera (Kyodo 2020).

El primer ministro Suga ha sido muy claro en manifestar su compromiso con Vietnam y con la región Indo-Pacífica, lo que se observa en sus declaraciones: "Japón, como nación del Indo-Pacífico, continuará contribuyendo a la paz y la estabilidad en esta región. Elegí Vietnam porque es el destino más adecuado para enviar este mensaje al mundo. El primer ministro Nguyen Xuan Phuc y yo hemos intercambiado muchas ideas sobre las relaciones bilaterales y cómo afrontar la covid-19”, dijo (Anh 2020). Otro ejemplo de que Suga dice las cosas claramente es cuando refirió que "desafortunadamente en esta región se han producido acontecimientos contrarios al Estado de derecho y a la apertura respaldados por las perspectivas de la ANSEA en el Mar de China Meridional. Japón se opone firmemente a cualquier acción que aumente las tensiones en el Mar de China Meridional” (Heydarian 2020a).

\section{RECUENTO SOCIOECONÓMICO 2020}

En 2020 ninguna sociedad o economía escapó de padecer efectos negativos como resultado de la pandemia de covid-19. Algunos ejemplos son la pérdida de empleo de personas vulnerables, bajos rendimientos productivos, cierre de actividades de todos los sectores, caída de exportaciones e importaciones, etcétera.

En relación con el empleo, según revela la Encuesta de Población Activa de Vietnam 2020, los trabajadores siguen siendo afectados por la pandemia, y las autoridades de gobierno plantearon tres recomendaciones a seguir: 1) Continuar con la implementación de los paquetes de ayuda financiera especificados la Resolución 42/NQ-CP del gobierno de manera oportuna, eficiente y correcta. Centrarse en el apoyo a varios grupos de trabajadores, informales, formales, en empresas y entidades productivas y comerciales. 2) Realizar investigaciones para diseñar paquetes de apoyo especiales específicamente para grupos vulnerables, incluidas trabajadoras, personas sin calificaciones técnicas o profesionales y trabajadores informales, para que puedan encontrar trabajo rápidamente y contribuir con su parte a la recuperación y el desarrollo económicos. 3) Fortalecer políticas habilitadoras en 
apoyo de empresas y entidades productivas y comerciales para recuperar actividades económicas en todos los sectores industriales, en especial aquellos significativamente afectados por la covid-19 (GSO 2020a). Los ingresos, el trabajo y el empleo se vieron afectados; el alto nivel de desempleo y la tasa de subempleo ponen en riesgo la seguridad social.

Obviamente, todos los sectores productivos fueron afectados también por la pandemia. Uno de los más castigados es el turístico y el país sigue sin abrir sus fronteras al turismo. Sí tiene puertas abiertas a ciudadanos empresarios o estudiantes vietnamitas, pero con una revisión minuciosa a su llegada.

De enero a septiembre el PIB aumentó $2.12 \%$ en todos los campos socioeconómicos, la economía del país mantuvo relativamente crecimiento positivo, mostrando la relevancia y precisión en la dirección y gestión para la recuperación económica, prevención y determinación de enfermedades, y unanimidad de todo el sistema político. Algunas claves socioeconómicas de enero a septiembre de 2020 son positivas (GSO 2020b):

Producto interno bruto (PIB): $+2.12 \%$

Índice de producción industrial (IIP): $+2.4 \%$

Número de empresas registradas recientemente: 98954 empresas

Ventas minoristas de bienes y servicios: $+0.7 \%$

Inversión: $+4.8 \%$

Exportación total de bienes: $+4.2 \%$

Importación total de bienes: $-0.8 \%$

Excedente comercial: 16.99 USD

Visitantes internacionales a Vietnam: $-70.6 \%$

Índice de precios al consumidor: $+3.85 \%$

Inflación subyacente: $+2.59 \%$ 
La industria manufacturera, aunque no creció mucho, siguió siendo el motor del crecimiento económico de enero a septiembre. La oferta y la demanda internas estaban garantizadas, la compra y el consumo de las personas aumentaron nuevamente. La balanza comercial de bienes alcanzó un superávit comercial. El avance del desembolso de la inversión pública mejoró de manera notable. Sin embargo, la economía y la sociedad siguieron enfrentando desafíos, especialmente económicos. A pesar de que la pandemia se pudo controlar, la propagación en el mundo fue muy complicada, y provocó la interrupción de las cadenas de suministro globales, con afecciones en el comercio, el turismo, el transporte y las industrias de importación y exportación, escasez de materiales de producción en las empresas, y reducción y cierre de operaciones.

Externamente, la guerra comercial entre Estados Unidos y China allanó un poco el camino para ampliar el comercio de Vietnam. Grandes firmas tecnológicas buscaron aumentar sus operaciones de manufactura en Vietnam, y, como comenta Hutt, esto elevó las ambiciones de un país que ya estaba en camino a convertirse en un potente fabricante de teléfonos y otros dispositivos de lujo. (Hutt 2020a). Apple, Nintendo y Foxcom (electrónicos de Taiwán) fueron algunas empresas que buscaron lugares de bajos salarios donde fabricar o terminar sus productos. Estas empresas consideraron a Vietnam como lugar donde establecerse. En mayo se informó que Apple comenzó a producir en Vietnam entre 3 y 4 millones de unidades, esto es, 30\% de sus auriculares AirPod, señal de que la empresa está trasladando algunas de sus cadenas de suministro de China a Vietnam (Hutt 2020b). Los salarios mínimos en Vietnam son más bajos incluso que en vecinos más pobres como Camboya, y actualmente oscilan entre \$132 y \$190 por mes, según la ubicación (Hutt 2020b).

De lo anterior, no se trata de decir que Vietnam supere a China porque existen diferencias muy importantes. Por ejemplo, China tiene alrededor de 800 millones de trabajadores manufactureros, mientras que Vietnam tiene 55 millones; en producción manufacturera y en manejo de contenedores no hay comparaciones. Lo que sí resulta factible es que la economía vietnamita pueda recuperarse más rápido que la de otros países del Sureste Asiático en 2021, especialmente si países como Estados Unidos, Japón y la Unión Europea movilizan sus cadenas de suministro pospandémicas de China y las colocan en Vietnam. 
En suma, los aspectos positivos en política exterior que en 2020 dieron prestigio internacional a Vietnam fueron la doble asignación, como presidente de ANSEA y como miembro no permanente en el Consejo de Seguridad de Naciones Unidas, que supo atender con acierto en medio de la pandemia. Asimismo, la firma del Acuerdo de Libre Comercio con la Unión Europea, reconocido por el Consejo Europeo como el acuerdo más ambicioso que se haya firmado con un país en vías de desarrollo, dio cierto prestigio como negociador y productor de bienes de calidad. Aunque no fue tema de este reporte, Vietnam fue reconocido por haber controlado el contagio de la covid-19, experiencia que compartió en los espacios de las Naciones Unidas y de la ANSEA.

Los retos por superar se encuentran en los problemas ambientales y los efectos del cambio climático que afectan una de las regiones más productivas, por ejemplo el delta del Mekong. Se hicieron esfuerzos por llevar el tema a la mesa de discusiones, pero pesó más el efecto de la pandemia. De igual forma, el asunto eterno del reclamo territorial del Mar del Sur de China Meridional, tan sólo mencionado por la serie de notas verbales emitidas por reclamantes y no reclamantes, quedó, nuevamente, sin solución. Es factible que, del recuento, Vietnam tenga un futuro prometedor en los ámbitos político, económico y social, porque se observa el trabajo laborioso y exitoso que realizó a contracorriente.

Maricela Mireya Reyes López es doctora en Estudios de Asia y África con especialidad en Sureste de Asia por El Colegio de México. Realizó estudios de maestría en la Universidad de Colima y de licenciatura en Relaciones Internacionales en la Universidad Femenina de México.

mar@ucol.mx

\section{REFERENCIAS}

Amnistía Internacional. s. f. "Vietnam.” Amnistía Internacional. https://www.es.amnesty. org/en-que-estamos/paises/pais/show/vietnam/ 
Anh, Viet. 2020. “Japan's Suga pledges immediate disaster assistance to Vietnam." VnExpress, octubre 19, 2020. https://e.vnexpress.net/news/news/japan-s-sugapledges-immediate-disaster-assistance-to-vietnam-4178909.html

ASEAN Vietnam. s. f. “Cohesive and Responsive ASEAN". ASEAN Vietnam 2020. https:// www.asean2020.vn/web/asean_en/gioi-thieu-ve-asean-2020

Bateman, Sam. 2020. “Demystifying Australia’s South China Sea stance.” East Asia Forum, agosto 12, 2020. https://www.eastasiaforum.org/2020/08/12/demystifying-australiassouth-china-sea-stance/

Brown, David. 2020. "Economic Reality May End More Big Mekong Dams.” Asia Sentinel, julio 1, 2020. https://www.asiasentinel.com/p/threat-of-further-big-dams-on-the

Diep, Vo Ngoc. 2020. "Viet Nam's Note Verbale No.22/HC-2020: A Commentary." Maritime Issues, mayo, 7, 2020. http://www.maritimeissues.com/law/viet-namsnote-verbale-no22hc2020-a-commentary.html

Fabry, Elvire. 2020. "RCEP: The Geopolitical Impact from a New Wave of Economic Integration." Jacques Delors Institute, diciembre 2, 2020. https://institutdelors. eu/en/publications/rcep-the-geopolitical-impact-from-a-new-wave-of-economicintegration/

Fawthrop, Tom. 2020. "The Last Farewell to the Mighty Mekong." The Diplomat, septiembre 2, 2020. https://thediplomat.com/2020/09/the-last-farewell-to-themighty-mekong/

GSO. 2020a. "Press Conference to Announce the Report on Labour and Employment Situation of the Third Quarter and 9 Months of 2020." General Statistics Office of Vietnam, octubre 6, 2020. https://www.gso.gov.vn/en/data-and-statistics/2020/10/ press-conference-to-announce-the-report-on-labour-and-employment-situation-ofthe-third-quarter-and-9-months-of-2020/

GSO. 2020b. "Press Conference to Announce the Report on Social and Economic Situations in the Third Quarter and 9 Months of 2020." General Statistics Office of Vietnam, septiembre 29, 2020. https://www.gso.gov.vn/en/data-and-statistics/2020/10/press- 
conference-to-announce-the-report-on-social-and-economic-situations-in-the-thirdquarter-and-9-months-of-2020/

GSO. 2020c. "Socio-Economic Situation in the Second Quarter and Six Months of 2020." General Statistics Office of Vietnam, junio 29, 2020. https://www.gso.gov.vn/en/dataand-statistics/2020/07/socio-economic-situation-in-the-second-quarter-and-the-first6-beginning-months-of-2020/

Haver, Zachary. 2020. "Sansha and the Expansion of China's South China Sea Administration." The Asia Maritime Transparency Initiative, Center for Strategic and International Studies (CSIS), mayo 12, 2020. https://amti.csis.org/sansha-and-theexpansion-of-chinas-south-china-sea-administration/

Heydarian, Richard Javad. 2020a. "ASEAN finally pushes back on China's sea claims.” Asia Times, junio 30, 2020. https://asiatimes.com/2020/06/asean-finally-pushes-back-on$\underline{\text { chinas-sea-claims }}$

Heydarian, Richard Javad. 2020b. "Suga launches charm offensive in Southeast Asia." Asia Times, octubre 23, 2020. https://asiatimes.com/2020/10/suga-launches-charmoffensive-in-southeast-asia

Hoang, Ha Hai. 2020. "Understanding the EU-Vietnam Free Trade Agreement.” East Asia Forum, mayo 21, 2020. https://www.eastasiaforum.org/2020/05/21/understandingthe-eu-vietnam-free-trade-agreement/

Hutt, David. 2020a. “US, Vietnam ties have never been better.” Asia Times, julio 13, 2020. https://asiatimes.com/2020/07/us-vietnam-ties-have-never-been-better/

Hutt, David. 2020b. "Why 'Made in Vietnam' won't replace 'Made in China'." Asia Times, mayo 21, 2020. https://asiatimes.com/2020/05/why-made-in-vietnam-wont-replacemade-in-china/

Huyền, Ánh y Lê Phương. 2020. "Vietnam reafirma su papel proactivo en el Consejo de Seguridad de la ONU." La Voz de Vietnam, julio 8, 2020. https://vovworld.vn/esES/enfoque-de-actualidad/vietnam-reafirma-su-papel-proactivo-en-el-consejo-deseguridad-de-la-onu-878667.vov 
Ketelsen, Tarek, Timo Räsänen y John Sawdon. 2020. "Did China turn off the Lower Mekong? Why data matters for cooperation.” Southeast Asia Globe, mayo 13, 2020. https://southeastasiaglobe.com/china-mekong-river-flow/

Koema. 2020. "Demographic Statistics of Vietnam." Julio, 2020: https://knoema.es/ VNDEM2018/demographic-statistics-of-vietnam?region=1000570-mekong-delta

Kyodo. 2020. "Suga and Vietnamese PM meet, with focus on economic and defense cooperation." The Japan Times, octubre, 19, 2020. https://www.japantimes. co.jp/news/2020/10/19/national/politics-diplomacy/vietnam-japan-yoshihide-suga/

Nguyen, Phuong y Neil Jerome Morales. 2020. "Vietnam, Philippines denounce China military drills in disputed waters." Reuters, julio 2, 2020. https://world.einnews. com/article_detail/520834774/Wf142xggwtlQi3I6

Nhân Dân. 2020. “ONU reconoce grandes avances de Vietnam en materia de derechos humanos." Nhân Dân, septiembre 20, 2020. https://es.nhandan.com.vn/sociedad/ item/1590780-onu-reconoce-grandes-avances-de-vietnam-en-materia-de-derechoshumanos.html

Osborne, Zoe. 2020. “The great salt drought desiccating Vietnam's Mekong Delta.” Aljazeera, abril 22, 2020. https://www.aljazeera.com/features/2020/4/22/the-greatsalt-drought-desiccating-vietnams-mekong-delta

Parlamento Europeo. 2020. “Aprobados los acuerdos de libre comercio y protección de inversiones UE-Vietnam." Noticias Parlamento Europeo, febrero 12, 2020. https://www.europarl.europa.eu/news/es/headlines/priorities/globalizacion/2020020 $\underline{6 \text { IPR72012/aprobados-los-acuerdos-de-libre-comercio-y-proteccion-de-inversiones- }}$ $\underline{\text { ue-vietnam }}$

Quy, Nguyen. 2020. "Northern Vietnam suffers longest heat wave in 49 years." VnExpress International, julio 20, 2020. https://e.vnexpress.net/news/news/northern-vietnamsuffers-longest-heat-wave-in-49-years-4133038.html 
Tran, Bich T. 2020. "Vietnam leads ASEAN through Covid-19." East Asia Forum, junio 2, 2020. https://www.eastasiaforum.org/2020/06/01/vietnam-leads-asean-through-covid$\underline{19 /}$

VietNamNet. 2017. "Economists say Vietnam focuses too much on rice exporting." Mekong Eye, octubre 20, 2017. https://www.mekongeye.com/2017/10/10/economists-sayvietnam-focuses-too-much-on-rice-exporting/

Zhou, Laura. 2020. "Mekong nations face growing threat to food security amid claims China's dams exacerbate effects of drought." South China Morning Post, mayo 10, 2020. https://www.scmp.com/news/china/article/3083523/mekong-nations-facegrowing-threat-food-security-amid-claims-chinas-dams 